\title{
Annotation
}

\section{Will exogenous surfactant treat respiratory distress syndrome?}

Since the late 1960s, when it became clear that one factor contributing to neonatal respiratory distress syndrome (RDS) was abnormal pulmonary surfactant on the alveolar surface, there has been the tantalising thought that it might be possible to prevent or at least ameliorate this serious condition by administering exogenous surfactant to the babies' lungs after birth.

\section{RDS is not simply due to surfactant deficiency}

Before an effective exogenous surfactant can be developed and delivered, a detailed knowledge is required of the biochemistry, biophysics, and physiology of pulmonary surfactant. During the years a great deal of knowledge about surfactant has accumulated, but important gaps remain. It is well known that babies who develop RDS have reduced levels of surfactant, and that the composition of this surfactant is abnormal (compared with surfactant from mature lungs) - that is the lecithin is more unsaturated and the phosphatidylglycerol levels low. However, the composition of surfactant is a complex mixture of many other phospholipids with very varied fatty acid chains together with cholesterol, triglycerides, free fatty acids, and proteins. This composition varies with the technique of harvesting and analysing the surfactant and we still do not know the exact composition of surfactant when it is spread on the alveolar surface, particularly as the composition may change between inspiration and expiration. The contribution of most of these substances to the physical properites and physiological functions of surfactant is still unknown. However it is apparent that slight changes in this composition have profound influence on the physical properties. The details of these subtle interactions remain to be fully demonstrated.

Although surfactant is reduced and abnormal in RDS, it is only one of many factors which is immature in preterm babies with RDS. Their lung structure is poorly developed ${ }^{1}$ with alveoli that are only just changing from terminal air spaces and bronchial branches which are far fewer than those of mature babies and are often still lined with immature epithelium. Between the alveoli there is still a large amount of interstitial tissue hindering the effective diffusion of gases. These problems are made worse by the epithelium's permeability to proteins which leak on to the lung's surface and interfere with the actions of surfactant. The preterm lungs are also inefficient at clearing lung fluid and its persistence after delivery contributes to the baby's respiratory difficulties. The preterm baby's ability to breathe is reduced by the poorly developed respiratory control and respiratory muscles, so that respiratory efforts may be inefficient, leading to underventilation of the lungs. This is complicated by immaturity of the pulmonary blood vessels with problems of either pulmonary vasoconstriction after hypoxia and hypercarbia or, conversely, of overperfused and oedematous lungs as a consequence of a patent ductus arteriosus.

\section{Experience with natural surfactant treatment}

With due regard to the complex nature of preterm babies' lungs, and their surfactant abnormalities, several research groups have tried to instil exogenous surfactants into lungs which are deficient in surfactant. These experiments can be divided into two groups. One group uses natural surfactant (that is whole lung surfactant with all its component parts), the other has constructed an artificial surfactant which has physical properties similar to the properties of natural surfactant, but of a completely different composition.

The work using natural surfactant was pioneered by Enhörning and Robertson ${ }^{2} 3$ who demonstrated in a series of experiments that when exogenous surfactant was obtained by concentrating saline lavages of mature rabbits' lungs, it could be instilled into the lungs of preterm 27-day fetal rabbits with considerable benefit. It improved lung expansion, oxygenation, compliance, and survival. It also led to a reduction in the pressure needed to ventilate the animals and prevented the development of cellular necrosis and hyaline membrane formation. ${ }^{4}$ Subsequently, this technique has been used to great effect in lambs ${ }^{5}$ and monkeys. ${ }^{6}$ This work is impressive in 
demonstrating that exogenous natural surfactant can improve most aspects of lung function which are abnormal in RDS. However, as with all experiments on animals it carries the caveat that 27-day rabbits or preterm lambs are not necessarily the same as preterm human babies, particularly with regard to a disease as complex as RDS.

Recently, this work has been extended by a small study on human babies conducted in Toronto using purified natural surfactant from calf lungs. The results were encouraging, in that overall they showed a transient but satisfactory improvement in the babies' blood gas status. However, they were made difficult by the problem that before the exogenous surfactant was given the babies had severe and established RDS, with all its complicating factors. ${ }^{7}$

Hallman has reported some interesting preliminary studies on the effects of treating 5 established cases of RDS with a single dose of $70 \mathrm{mg} / \mathrm{kg}$ of exogenous natural surfactant extracted from human amniotic fluid. ${ }^{8}$ In these babies there was improvement in blood gases which was short lived although the subsequent course of the disease was milder than expected.

Fujiwara et al. ${ }^{9}$ used a surfactant that was a mixture of natural and artificial components. They used minced bovine lung lipids and then added dipalmitoylphosphatidylcholine and phosphatidylglycerol to improve the physical properties. When this mixture was instilled as a suspension into the trachea of the 10 babies with established RDS, the babies had a very rapid improvement in their blood gas status and oxygen requirements. Unfortunately, a high proportion developed problems from a patent ductus arteriosus. Lamb experiments with natural lung surfactant have also shown a high incidence of patent ductus arteriosus. ${ }^{10}$ Whether this was a consequence of the administered surfactant is, as yet, unclear. This is a worrying side effect which may be due to a component of the natural surfactant or the fluid used in the instillation. It has not been seen in babies treated with artificial surfactant.

\section{Artificial lung surfactant}

Natural surfactant, or enhanced natural surfactants, would appear to be potentially effective exogenous surfactants. However their potential for widespread use is limited. Firstly, it is difficult to obtain sufficient natural surfactant to treat all those babies requiring it. Secondly, the surfactant may be contaminated by extraneous proteins or pathogens, and so it would have to be submitted to rigorous purification and control, which would result in expense and further losses of the precious material.

In view of this, several groups have been interested in trying to produce artificial lung surfactants. These do not necessarily have to be made from the components of natural lung surfactant. Several different mixtures of substances may well be effective provided they have the appropriate physical properties and are not toxic to the preterm lung. To be effective, an exogenous lung surfactant should spread to cover the air or water interface with a complete monomolecular layer which reduces the contractile force of the water. It must be able to maintain and replenish this monolayer even if the surface is being expanded, compressed, or distorted. If the monolayer is compressed it must be able to withstand the forces without buckling, resisting a surface pressure in excess of $72 \mathrm{mN} / \mathrm{m} .11$

At present, it appears that the major surfactant component dipalmitoylphosphatidylcholine is important in any surfactant mixture because the shape of the molecules allow them to self assemble into a stable surface monolayer. When this is compressed the molecules pack together and solidify so that it is able to resist forces in excess of 100 atmospheres. It is this property which is vital in preventing alveolar collapse. Other components are needed in the mixture to provide the rapid spreading characteristics; it is possible that several substances could provide this property. Bangham et al..$^{12}$ have produced an artificial lung surfactant fulfilling these properties which is made of pure dipalmitoylphosphatidylcholine with unsaturated phosphatidylglycerol as the spreading agent. In their initial clinical trial ${ }^{13}$ one dose of $25 \mathrm{mg}$ was given as a dry powder insufflated down the endotracheal tube, and the results were encouraging showing a considerable reduction in mortality and the need for less treatment to control the lung disease.

Other groups are engaged in synthesising and testing artificial lung surfactant mixtures but as yet all the results are tantalisingly preliminary.

\section{Problems still to be resolved}

There are still many questions to be answered and resolved before an effective exogenous surfactant will be available for routine clinical use. There remain differences of opinion about the best physical properties of an exogenous surfactant and the correct substances to produce it. It is hotly debated whether it should be delivered as a powder, a suspension, or a solution. At the moment no satisfactory technique exists for delivering exogenous surfactant consistently and noninvasively into the lungs of babies straight after birth. There are inadequate data on the appropriate dose or intervals between doses which would be required in babies of different sizes. Little is known about the interaction of exogenous surfactant with endogenous surfactants, 
nor the effect it might have on the synthesis or secretion of surfactant.

RDS is a complex disease with many compounding factors, of which inadequate surfactant is the major problem. Many studies during the last few years show it is likely that treatment with some form of exogenous surfactant will reduce the level of treatment needed for the disease. Exactly which surfactant preparation will finally be used and how effective it will prove to be remains to be elucidated.

\section{References}

1 Stahlman M T, Gray M E. Anatomical development and maturation of the lungs. Clin Perinatol 1978; 5: 181-96.

2 Enhörning G, Robertson B. Lung expansion in the premature rabbit fetus after tracheal deposition of surfactant. Pediatrics $1972 ;$ 50: 58-66.

3 Enhörning G, Grossmann G, Robertson B. Pharyngeal deposition of surfactant in the premature rabbit fetus. Biol Neonate 1973; 22: 126-32.

4 Nilsson R, Grossmann G, Robertson B. Lung surfactant and the pathogenesis of neonatal bronchiolar lesions induced by artificial ventilation. Pediatr Res 1978; 12: 249-55.

5 Adams F H, Towers B, Osher A B, Ikegami M, Fujiwara T, Nozaki M. Effects of tracheal instillation of natural surfactant in premature lambs. I. Clinical and autopsy findings. Pediatr Res 1978; 12: 841-8.

6 Enhörning G, Hill D, Sherwood G, Gutz E, Robertson, B, Bryan C. Improved ventilation of prematurely delivered primates following tracheal deposition of surfactant. Am J Obstet Gynecol 1978; 132: 529-36.

${ }^{7}$ Bryan C. In: Raivio K O, ed. Sigrid Juselius Foundation Conference on the Respiratory Distress Syndrome, Helsinki, August 1982. New York: Academic Press, 1983: in press.

8 Hallman M. In: Raivio K O, ed. Sigrid Juselius Foundation Conference on the Respiratory Distress Syndrome, Helsinki, August 1982. New York: Academic Press, 1983: in press.

9 Fujiwara T, Maeta H, Chida S, Morita T, Watabe Y, Ade T. Artificial surfactant therapy in hyaline membrane disease. Lancet 1980; i: 55-9.

10 Clyman R I, Jobe A, Heymann M, et al. Increased shunt through the patent ductus arteriosus after surfactant replacement therapy.J Pediatr 1982; 100: 101-7.

11 Morley C J, Bangham A D. Physical properties of surfactant under compression. In: von Wichert $\mathbf{P}$, ed. Clinical importance of surfactant defects. Progress in Respiration Research. Vol. 15. Basel: Karger, 1981 : 261-8.

12 Bangham A D, Morley C J, Phillips M C. The physical properties of an effective lung surfactant. Biochim Biophys Acta 1979; 573: 552-6.

13 Morely C J, Bangham A D, Miller N, Davis J A. Dry artificial lung surfactant and its effect on very premature babies. Lancet 1981 ; i: 64-8.

COLIN J MORLEY Department of Paediatrics, Level 8, Addenbrooke's Hospital, Hills Road, Cambridge CB2 $2 Q Q$

\section{Notice to contributors}

Please note that revised guidelines for the submission of manuscripts have been printed inside the front cover of this journal and, in particular, that two copies of papers should be submitted. 